\title{
DESPLAZAMIENTO Y AISLAMIENTO. ALIENADOS MENTALES EN LA CIUDAD DE MEDELLÍN. 1878-1930*
}

\author{
Álvaro León Casas Orrego \\ Universidad Pablo de Olavide - Universidad de Antioquia
}

\section{RESUMEN}

La observación sobre la ciudad de Medellín en el contexto de la historia urbana, generó un sorprendente cuadro de temas sobre higiene y salud pública, entre los que encontramos el caso de los alienados mentales y los dispositivos de control propuestos por las autoridades civiles y los médicos. De 1880 hasta 1950 Medellín vivió el proceso de modernización, que la convirtió en polo de atracción de los desplazamientos de población al interior de la provincia de Antioquia. El Ferrocarril garantizó desde los pueblos vecinos la movilización masiva de población, entre la que llegaron no pocos alienados mentales. A finales del siglo XIX, las autoridades crearon la Casa de Alienados para dar asilo a estas personas, esta institución se convirtió a comienzos del siglo XX en Manicomio Departamental y a mediados del siglo XX en el Hospital Mental de Antioquia. El aislamiento de los locos da cuenta del comienzo del proceso de constitución e institucionalización del saber psicopatológico, de la autoridad médica y la medicalización de la demencia en Antioquia a comienzos del siglo XX.

PALABRAS CLAVE: Desplazamiento. Alienación. Aislamiento. Manicomio. Enfermedad mental.

\section{DISPLACEMENT AND ISOLATION. MENTAL ILLNESS IN THE CITY OF MEDELLIN. 1878-1930}

\begin{abstract}
The observation in the city of Medellín within the framework of urban history generated a surprising picture of subjects in hygiene and public health, among which we found the case of mental illness and the control systems proposed by civilian authorities and doctors. In Medellín, between 1880 and 1950 , the modernization process went on, turning its pole of attraction on population
\end{abstract}

* Este artículo se inscribe en la línea de investigación historia de la medicalización e higiene, del Grupo Historia de la Salud de la Universidad de Antioquia y la Universidad Nacional de Colombia sede Medellín. 
displacements to the interior of the province of Antioch. As a result of Railroad I, there was a massive mobility of population from the neighboring towns, including some mentally ill people. At the end of the 19th century, the authorities created a house of asylum for these people, which became the Mental Hospital in the middle of the 20th century. The isolation of the mentally ill people reports both medical authority and the beginning of the constitution process and institutionalization of the psychopathology and medicalization of mental diseases in Antioquia at the beginning of the 20th century.

KEY WORDS: Displacement. Alienation. Isolation. Mental hospital. Mental illness.

\section{LA CIUDAD}

Un estudio de historia urbana realizado sobre la ciudad de Medellín ${ }^{1}$, reveló una interesante observación sobre la dinámica de formación espacial urbana moderna, que entre otros actores, involucró a población desplazada desde los municipios vecinos y la presencia en las calles de la ciudad de los llamados alienados mentales. Las autoridades civiles implementaron dispositivos de regulación que comenzaron en la creación de la Casa de Alienados Mentales en 1873, institución que significó el comienzo de lo es hoy la E.S.E. Hospital Mental de Antioquia HOMO.

La Villa de la Candelaria de Medellín ${ }^{2}$ desde su fundación en 1670, vivió una particular dinámica de su economía que la convirtió desde el siglo XIX en el primer centro económico de la provincia, en competencia con la ciudad de Santafe de Antioquia, primera capital de la provincia y la ciudad de Santiago de Arma de Rionegro, cardinal centro comercial ubicado al oriente, en la ruta comercial del río Magdalena, que conectaba a la provincia de Antioquia con el resto del Virreinato de La Nueva Granada. La primacía comercial que la Villa de Medellín había alcanzado desde finales del siglo XVIII ${ }^{3}$, fortaleció a una clase social de comerciantes, mineros y terratenientes que acumularon las riquezas suficientes para lo que sería el despegue industrial de la

1 Cfr. Botero, F. (1987), Medellin, 1890-1950. Historia urbana y juego de intereses, Medellín, Universidad de Antioquia.

2 Antiguo nombre de Medellín, actualmente segunda ciudad en importancia económica y política de Colombia. Con vocación comercial, industrial y financiera, La ciudad alberga actualmente más de dos millones de habitantes: Departamento Administrativo de Planeación de Medellín. Unidad de Clasificación Socioeconómica y Estratificación, 2005.

3 Cfr. CASAs, Á. (1985), El comercio en las ciudades de Antioquia, Medellín, Universidad de Antioquia, Tesis, (director Víctor Álvarez M.), p. 98. 
ciudad a comienzos del siglo $\mathrm{XX}^{4}$. El espíritu pragmático y emprendedor de los antioqueños ${ }^{5}$ de Medellín los condujo a convertir la villa en ciudad y en capital de la provincia de Antioquia en el año 1830. A partir de esa fecha, la actividad comercial se ve acompañada por la actividad política y administradora. La ciudad es desde entonces la sede de uno de los poderes regionales más destacados en la historia colombiana de los siglos XIX y XX'.

En el proceso de modernización de las ciudades en Colombia, entre $1880 \mathrm{y}$ 1940 aproximadamente, las autoridades civiles de la ciudad de Medellín, apremiadas por la necesidad de tener un control sobre la creciente población que en ella se albergaba e incidir sobre las transformaciones urbanas, generaron simultáneamente el despliegue de iniciativas privadas y proyectos municipales que tenían como una de sus bases más destacadas, la intervención en el proceso de desplazamiento de población hacia la capital y los consecuentes problemas de higiene y salubridad que ellos provocaron a nivel urbano.

La concentración económica, política y demográfica en la ciudad de Medellín, produjo en la segunda mitad del siglo XIX, la administración de estrategias de control social y urbano. Las autoridades civiles buscaron dar solución a problemas de ordenamiento de la ciudad para que ésta se adaptara al desarrollo económico y crecimiento demográfico que causó estrechez en el uso de los espacios, con la consecuente carencia de viviendas y establecimientos para el comercio y la industria. En el transcurso de la segunda mitad del siglo XIX la población de Medellín se triplicó. De 17.644 que sumaba la población rural y urbana en 1851, paso a 59.815 en 1905, lo que significó un crecimiento anual promedio entre el 2 y 3 por ciento. La concentración de habitantes en la ciudad de Medellín en ese mismo periodo, se ha calculado con una oscilación entre $38.5 \%$ y $56.8 \%$, en relación con la población del Valle de Aburrá7, entre 1851 y 1905; y una oscilación entre un $18.8 \%$ en

4 Sobre consolidación de la industria en Medellín ver Botero, F. (1985), La industrialización en Antioquia: génesis y consolidación 1900-1930, Medellín, Universidad de Antioquia, Centro de Investigaciones Económicas, p. 15.

5 Sobre el origen de la idea del espíritu emprendedor asociado a la mentalidad religiosa de los antioqueños, cf. FAJARDO, L.H. (1966), La moralidad protestante de los antioqueños, Cali.

6 Cf. Uribe, M.T. y ÁlvareZ, G.J. (1987), Poderes y regiones en la Constitución de la nación colombiana, Medellín, Editorial Universidad de Antioquia.

7 El Valle de Aburrá, corresponde a las tierras que se extienden a lado y lado del río Medellín, en forma longitudinal, de sur a norte, en donde estaban asentadas en la segunda mitad del siglo XIX siete poblaciones importantes, que hoy forman el ente administrativo Área Metropolitana del Valle de Aburrá. Hermelin, M. (1996), Geología y paisaje. En Melo, J. (ed.), Historia de Medellín I, Medellín, Suramericana de Seguros, pp. 3-16. 
1851 y un $15.9 \%$ en relación con la población del Departamento de Antioquia ${ }^{8}$ en ese mismo periodo ${ }^{9}$.

La aglomeración y los amontonamientos densamente concentrados en los límites de la ciudad que conservaba en lo fundamental la estructura de los poblados y villas coloniales, asfixiaban a los habitantes en un ambiente malsano, cuya característica principal la constituía la falta de un sistema moderno de abastecimiento de agua potable domiciliaría, una red de evacuaciones subterráneas de las aguas servidas, la recolección de basuras, barrido de calles y separación de los espacios que determinan las diferentes practicas económicas y sociales, y la presencia de vagos, mendigos y alienados mentales en las calles de la ciudad. En este sentido en los registros documentales oficiales de los archivos locales, el historiador puede observar que el tema de la salubridad de la ciudad constituyó uno de las principales motivaciones en el diseño de políticas públicas de salud, de iniciativa pública o privada.

En el presente artículo se quiere mostrar una de las consecuencias de ese desplazamiento de población, desde los pueblos hacia la capital del departamento de Antioquia; aquella que afectó a algunas personas en su salud mental, haciéndolas parecer alienadas mentales. También, hacer una breve descripción de las circunstancias en las que se produjo ese desplazamiento de población, los efectos en la población residente en la ciudad, así como las acciones y los dispositivos de control y aislamiento que las autoridades civiles y médicas implementaron para hacer frente a ese fenómeno que a comienzos del siglo XX hizo presencia en Medellín en su proceso de modernización.

\section{EL DESPLAZAMIENTO}

El movimiento de población desde los pueblos ruralizados hacia la capital, ha constituido una constante en la historia de Antioquia ${ }^{10}$, desde la primera mitad del siglo XIX. A finales de ese siglo, el médico francés Charles Safray,

8 La antigua provincia de Antioquia, constituye hoy el ente administrativo territorial de carácter Departamental, del cual Medellín es capital desde 1830.

9 Sobre crecimiento demográfico y concentración poblacional en la ciudad de Medellín cf. Álvarez, V. (1996), Poblamiento y población en el Valle de Aburrá y Medellín 15411951. En Melo, J. (ed.), pp. 57-84.

10 Cf. Villegas, L. (1993), Poblamiento y vida diaria en el nororiente de Medellín, 1900-1957, Medellín, Universidad Nacional de Colombia sede Medellín, Tesis, [s. n.]. Sobre el impacto del movimiento demográfico en Antioquia cf._LóPEZ, T.A. (1979), Migración y cambio social en Antioquia durante el siglo diez y nueve, Medellín, Hombre Nuevo. 
en su viaje por La Nueva Granada advirtió que «el comercio, la industria, la instrucción, en una palabra, toda la prosperidad de este país, depende tan sólo de una cosa: de los caminos» ${ }^{11}$. El obstáculo que para el desarrollo económico y social de los pueblos instalados en la región antioqueña representaba el estado de sus caminos, no era un problema nuevo. Éste se había hecho manifiesto desde el siglo XVIII, cuando en 1785 el juez y visitador Juan Antonio Mon y Velarde, en el contexto de la aplicación de las Reformas Borbónicas en Antioquia, intentó una amplia y estratégica política de apertura de caminos ${ }^{12}$. Otros estudios sobre el siglo XIX también han constatado que en el periodo republicano, hasta bien avanzada la segunda mitad de esta centuria, las reformas del juez visitador de Antioquia, no aportaron cambios significativos. Según Jorge Robledo, «(...) en 1870, las vías terrestres eran solamente caminos para peatones y bestias» ${ }^{13}$.

Sólo después de 1870, las soluciones de transporte por vía terrestre en Antioquia presentaron cambios verdaderamente significativos. La construcción del Ferrocarril de Antioquia ${ }^{14}$, como moderno medio de transporte, posibilitó mantener la dinámica agro-exportadora, pero al mismo tiempo se convirtió en vehículo de las personas que buscaban en la ciudad de Medellín mejorar sus condiciones de vida.

Ese desplazamiento de población hacia la capital del departamento, constituyó una paradoja del mismo crecimiento económico; ya que en la segunda mitad del siglo XIX, éste condujo la economía nacional a su inserción en el comercio mundial, adaptando la estructura de comunicaciones a la dinámica agro-exportadora. El crecimiento económico de Medellín aumentó con la incorporación de nuevas prácticas políticas, sociales y culturales que convirtieron a la ciudad en centro de atracción de pobladores de toda la provincia ${ }^{15}$. En general, la apertura de caminos que conducían al Valle de Aburrá coadyuvó a que llegaran a Medellín todo tipo de personas en busca de futuro. Para la

11 SAfray, C. (1948), Viaje a Nueva Granada, Bogotá, Biblioteca Popular de Cultura Colombiana, p. 205.

12 Cf. CASAS, Á. (1985), p. 43.

13 Robledo, E. (1954), Bosquejo bibliográfico del señor Oidor Juan Antonio Mon y Velarde Visitador de Antioquia 1785-1788, Bogotá, Banco de la República, citado por FERRO, G. (1994), p. 44.

14 BARnhart, D. (1977), Auge y fracaso: La historia de la construcción de los ferrocarriles. En Bejarano, J. (compilador) (1978), Siglo XIX en Colombia visto por historiadores norteamericanos, Bogota, La Carreta, p. 279.

15 Medellín se convierte además en la segunda ciudad de importancia en Colombia, con 29.765 habitantes, después de Bogotá con 40.883 en 1870. Cf. Álvarez, V. (1996), p. 75. 
segunda mitad del siglo XIX se concentraban en Medellín las actividades administrativas, económicas, políticas de todo el Departamento, lo mismo que los servicios educativos y de salud, y la beneficencia. El crecimiento económico y social de la ciudad la había transformado en un centro de convergencia de diversas actividades y al mismo tiempo en un punto de destino de diferentes líneas migratorias que llegaban a la capital del departamento de Antioquia. En particular, Medellín fue receptora durante el cambio del siglo XIX al XX de un flujo migratorio que no siempre encontró las posibilidades económicas de supervivencia. En consecuencia, entre estos inmigrantes se fue formando una masa de mendigos y desadaptados que serían el objeto de la caridad pública, de los programas de beneficencia de la municipalidad y de otras entidades de carácter privado como la Sociedad del Sagrado Corazón y la Sociedad San Vicente de Paúl ${ }^{16}$. Muchos de estos indigentes manifestaron formas de desequilibrio mental o de alienación, entendida aquí en su significado más amplio como extrañamiento de un individuo frente a otros, a la sociedad o al trabajo.

El uso de la expresión «alienados» mentales por parte de las autoridades civiles de Medellín en aquella época, es asociado por el historiador con los originales de los tratados de Pinel y Esquirol. Estos libros habían sido traducidos en España utilizando la palabra enagenación en lugar de la palabra alienation mentale utilizada por Pinel, y remplazando la expresión maladies mentales en el texto de Esquirol ${ }^{17}$. Por lo menos hasta 1960, esa expresión fue usada con frecuencia en los registros de historias clínicas del Hospital Mental de Antioquia, para referirse en general a los pacientes que allí ingresaban.

En la ciudad de Medellín a finales del siglo XIX, se consideraba alienado a aquel que no lograba insertarse en ninguna práctica social; al descodificado, al desinstitucionalizado, al que no encajaba en la norma y para el cual era necesaria la creación de una normativa especial; aquella que se instrumentalizaba para procurar el orden público, el código de policía. Desde finales del siglo XIX y hasta la primera mitad del siglo XX, el término hacía referencia a los trastornos mentales; se refería al hecho de que quien los padecía no era

16 Sobre instituciones de caridad y beneficencia en Medellín cfr. RUIZ, A. (1995), El discurso de las entidades de asistencia social en Medellín, 1890-1915, Medellín, Universidad de Antioquia, Tesis de antropología.

17 PINEL, P. (1800), Traité médico-philosophique sur làlienation mentale, Paris, Caile et Ranvier; ESQUIROL, J.E.D. (1838), Des maladies mentales considérees sous les rapports médical, hygiénique et médico legal, Paris, chez-Baillière. Referencia tomada de PLuMED J.J. (2005), La clasificación de la locura en la psiquiatría española del siglo XIX, Asclepio, 57 (2), p. 224. 
dueño de sí mismo, estaba fuera de sí, «no regía» su conducta, sino que actuaba tan contra sí mismo como si fuera otro quien tomara las decisiones por él o por ella ${ }^{18}$. Los registros de historias clínicas y las cartas de recomendación con que se remitían pacientes al Hospital, muestran que las instancias de delimitación de los estados de «alienación» eran diversas: podía ser el vecino, el esposo, el párroco, el arzobispo, el alcalde, el inspector de policía o un médico. La delimitación de la locura desde la psicopatología en Medellín será posterior a $1960^{19}$.

Sobre la manera en que las autoridades determinaban la necesidad del asilamiento de personas, Jorge Betancur enseña una interesante descripción sobre la presencia, en el céntrico barrio Guayaquil ${ }^{20}$, de inmigrantes con síntomas de alienación, actitudes mentales poco fiables o desequilibradas:

Como los volados de casa, como los pordioseros, como los buscadores de trabajo, como los busca fortunas, como las putas, también desquiciados y estúpidos hicieron de las calles de la ciudad su nuevo hogar. La figura de seres con rostros descompuestos, ropas ajadas y sucias, caminar lento y pesado, poco a poco se volvió familiar, en especial en sitios cercanos a la plaza de mercado. A menudo los guardias recogían a dementes de las calles aledañas y los llevaban a las inspecciones de policía ${ }^{21}$.

18 No se ignora aquí que desde diversas disciplinas se atribuyen al término otros significados: la psiquiatría considera que la alienación es un bloqueo auto inducido o una disociación de sentimientos que produce en la persona una reducción de su capacidad social y emocional con las consiguientes dificultades para ajustarse a la sociedad. Algunos filósofos creen que el origen de la alienación no está en la persona sino en una sociedad vacía y despersonalizada. Karl Marx, en su interpretación económica de la alienación, sostenía que las personas estaban alienadas de su propio trabajo, ya que al no poseer los medios de producción, otra persona (el propietario o capitalista) se apropiaba de su trabajo que pasaba a ser obligatorio y no creativo. Según Sigmund Freud, la alienación es un distanciamiento de uno mismo causado por la separación de las partes consciente e inconsciente de la mente. Para la sociología la anomia o desarraigo (postulada por Émile Durkheim) era la causa de la pérdida de la tradición social y religiosa. Posteriormente algunos sociólogos ampliaron la teoría de la alienación de Durkheim. Los existencialistas Sören Aabye Kierkegaard, Martin Heidegger y Jean-Paul Sartre consideraban que cierto grado de autoextrañamiento e impotencia ante el propio destino era algo consustancial a la condición humana (http://usuarios.lycos.es/) (24-07-08).

19 Cfr. Archivo Histórico del Hospital Mental de Antioquia (HOMO), Historias Clínicas, «Cartas de remisión»y «Recomendaciones», 1903-1987.

${ }^{20}$ Guayaquil es un barrio central de los más antiguos de la ciudad de Medellín, con la Estación Cisneros, donde terminaba la línea del Ferrocarril de Antioquia y funcionó el mercado público hasta el tercer cuarto del siglo XX.

21 La Defensa, Medellín, enero 8 de 1925, citado por Betancur, J. (2000), Moscas de 
De hecho esta calidad de inmigrantes en la ciudad de Medellín constituyó un sector social integrado por el alienado, el vago o el desadaptado, que fácilmente se asimilaba con el recién llegado. El ferrocarril cargó con el peso de lo que en Europa fueron en el siglo XV los Narrenschiff; barcos que transportaban a los locos que eran expulsados de una ciudad a otra y a los que Michel Foucault se refirió como "cargamentos insensatos», que eran detenidos por las autoridades municipales 22 . De hecho, el Ferrocarril de Antioquia contribuyó en gran medida al desplazamiento general de personas, pero evidentemente también a la llegada de alienados que eran remitidos por los alcaldes municipales de los pueblos hacia la capital. Por lo menos tres cuartas partes de las personas asiladas en el Hospital Mental entre 1903 y 1940, procedían de fuera del Valle de Aburrá y llegaban en tren, después de hacer varios transbordos en mula o a pie $^{23}$. En consecuencia, la emergencia de esa nueva realidad demográfica y poblacional, desde finales del siglo XIX y primera mitad del siglo XX, condujo a una especial preocupación oficial por los problemas de la salud pública en la ciudad de Medellín, y ésta cobijó las cuestiones del cuerpo y las de la mente.

La mayor presencia en las calles de personas alienadas que se confundían con mendigos y vagos, ocurrió en un momento en el que la densidad de población provocaba serias complicaciones de insalubridad e higiene ${ }^{24}$. El tema de la salud mental de los habitantes de la ciudad se fue convirtiendo en asunto de vital importancia para las autoridades civiles. Los archivos municipales abundan en registros sobre los inconvenientes causados por la aglomeración que generaba crisis de vivienda y locales para el comercio ${ }^{25}$. Las autoridades civiles consideraron necesario prestar atención y dar solución a esta situación. Sin embargo, otro poder ya tradicional, aunque todavía en proceso de formación, tendría la última palabra en un intento de solución del problema de la

todos los colores. Historia del barrio Guayaquil de Medellin 1894-1934, Bogotá, Ministerio de Cultura, p. 110.

22 «(...) la Nef des Fous, la nave de los locos, extraño barco ebrio que navega por los ríos tranquilos de Renania y los canales flamencos». FouCAULT, M. (1964), Histoire de la folie a l'age classique, Paris, Plon. Para este trabajo la versión en español de 1986, Historia de la locura en la época clásica, México, Fondo de Cultura Económica, p. 15.

23 De 2.212 asilados entre 1903 y 1940, solo 577 procedían de Medellín o poblaciones del Valle de Aburrá. Gomez, M. (2007), Sistematización del Archivo de historias clínicas, Archivo Histórico (HOMO), 1903-1964. (CD-ROM, inédito).

${ }^{24}$ Cfr. ReYes, C. (1996), Aspectos de la vida social y cotidiana de Medellín 1890-1930, Medellín, Tercer Mundo Editores, Premio Nacional de Cultura.

25 Cfr. Archivo Histórico de Medellín (AHM), Actas del Concejo, 1890-1950. 
presencia de personas consideradas dementes en las calles de la ciudad: la medicina y el cuerpo médico de la ciudad organizado en la Academia de Medicina de Medellín, fundada en 1887.

\section{EL AISLAMIENTO}

A finales del siglo XIX la medicina en Antioquia extendió su acción de medicalización al terreno de las enfermedades mentales aplicando medidas de exclusión, pero antes es «el código de policía el instrumento que contempla unas disposiciones para el secuestro de los «locos o dementes» que circulan por la ciudad» ${ }^{26}$. Claudia Montagut ha encontrado en los códigos de policía de 1878 y de 1914, disposiciones de control que unificaron criterios de acción frente a vagos, mendigos, dementes e indigentes. «Sobre el indigente y el loco - afirma - se procede de la misma manera, en la medida en que sobre ellos se tiene de inmediato poder absoluto de intervención para el cuidado del orden público» ${ }^{27}$. Siempre que aparecía en un lugar de la ciudad un individuo como indigente o loco ante la mirada pública, la policía procedía a su reconocimiento para asegurarlo. Si el individuo era forastero, el escrúpulo era más fuerte. Tal vez por esta razón los alrededores de la estación de trenes de Cisneros, en el barrio Guayaquil, era el lugar donde con mayor frecuencia se detenía a los sospechosos. Una escena cotidiana reportada por el historiador en 1925, debió repetirse de ordinario antes y después en aquel sector del centro de la ciudad, desde la llegada del Ferrocarril a la Plaza de Cisneros.

El jueves 26 de febrero de 1925 en las horas de la tarde, varias personas de la ciudad, de las que acostumbraban sentarse en las cercanías de la estación Cisneros del Ferrocarril de Antioquia a disfrutar la brisa en espera del tren, vieron bajar de uno de los vagones a un hombre extraño. Varios policías constataron su extravío, después de hacerle preguntas a las que respondió con incoherencias. No supo decir a los uniformados su nombre. Lo clasificaron como un nuevo demente que llegaba a la ciudad ${ }^{28}$.

La diferencia entre esta escena de 1925 y otra similar antes de 1878 , consistiría en la forma del aseguramiento o aislamiento de los individuos. Antes

26 Montagut, C. (1997), Formación del discurso psiquiatrico en Antioquia 1870-1930. Una cartografia de la exclusión, Tesis Universidad Nacional de Colombia sede Medellín, (director Alberto Castrillón), p. 140.

27 Montagut, C. (1997), p. 143.

28 BetAnCUR, J. (2000), p. 109. 
de 1878 el espacio de confinamiento era la cárcel municipal, mientras que a partir del segundo momento lo fue la Casa de Alienados Mentales, Hospital de Locos o Manicomio.

Algunos estudios sobre la locura han señalado que antes de 1878 la demencia era consideraba enfermedad del alma y no disfunción del cerebro ${ }^{29}$. Los indicios que se han reunido en la presente investigación indican que al parecer esta fue la concepción que de esa enfermedad se tuvo en Antioquia hasta la primera mitad del siglo XX, cuando se consideraba la alienación, en tanto que transgresión, objeto del discurso judicial. Sólo así se explica que la policía municipal, respaldada en el código, hubiese actuado como «instancia de delimitación». Con la creación en 1878 de la Casa de Alienados Mentales en Medellín, la delimitación de la locura comenzó su desplazamiento hacia la autoridad médica. Sin embargo, el código de policía no perdió vigencia; por el contrario, la conservó a favor de la determinación médica.

Diagnósticos como «delirio alcohólico» y «manía agresiva», a los que se agregaba en su mayoría la observación de «incurable», registrados en las historias clínicas del Hospital Mental de Antioquia por lo menos hasta 1960, asociaron al loco o alienado mental con el vago, el degenerado sexual, el alcohólico y hasta con el delincuente peligroso, al que era necesario separar temporal o definitivamente del cuerpo social, mediante el asilamiento ${ }^{30}$.

El hallazgo de obras de Freud, Farlet, Dieulafoy y Truosseau, entre los libros de la pequeña biblioteca que se formó en el Manicomio Departamental a partir de la llegada del médico Lázaro Uribe Calad a la dirección, hace pensar al psiquíatra Pedro Sánchez Gaviria en las tendencias que pudieron orientar el saber y la práctica de los médicos de Medellín en la primera mitad del siglo XX ${ }^{31}$. Sin embargo, psicólogos actuales del Hospital Mental de Antioquia, no han podido identificar en los diagnósticos de ingreso, antes de 1920, alguna nosología o convenciones médicas en las que éstas puedan encajar. Hasta 1930, la manía en sus diversas formas constituyó el diagnóstico más frecuente, asociado con agitación, delirio místico, melancolía, alcoholomanía, dipsomanía y alucinaciones auditivas. No obstante, la decisión del asilamiento fue policial y no clínica ${ }^{32}$.

En las primeras historias clínicas que se conservan en el Hospital Mental de Antioquia, según el examen realizado por Sánchez Gaviria, coordinador de

29 Pinel psicologista, referido por Plumed (2005), p. 225.

30 GOMEZ, M. (2007).

31 SANCHEZ, P.L. (.1995), Las primeras veinte historias clínicas del Hospital Mental de Antioquia, Revista Hospital Mental de Antioquia HOMO, (vol. \#), pp. 16-17.

32 Goméz, M. (2007), Hallazgos Clínicos, historias clínicas, HOMO, p. 4, (inédito). 
la consulta externa de dicho hospital en 1995, «no existe ninguna nota sobre fundamento de tipo clínico para formar el diagnóstico inicial de ingreso, ni para formar el diagnóstico final de baja»; es decir, que en esos registros «hay ausencia total de historia clínica». Hasta 1920, no se consignaban en la historia clínica los tratamientos empleados, fuesen éstos físicos o químicos ${ }^{33}$. La única terapéutica que allí se registraba, sobre todo hacia 1930 estaba asociada con enfermedades del cuerpo y no de la mente ${ }^{34}$.

En los códigos de policía de 1878 y 1914, que regían para la determinación del asilamiento en la Casa de Alienados y en el Manicomio, se observan diferencias en el tratamiento de los enajenados mentales. En 1878, lo que se buscaba con el loco era implementar los «medios para mantenerlo, proveer a su subsistencia y cuidado»; mientras que en 1914, se esperaba «seguridad»y, aunque no se especificaban las enfermedades, «procurar su curación» ${ }^{35}$; sin embargo, una vez ingresadas en el Hospital, a la mayoría de las personas se les considera «incurables» ${ }^{36}$.

La práctica pericial de loco, que hizo de la policía una instancia de delimitación de la locura, aparecía en los dos códigos con el único fin de determinar si se coartaba la libertad, como forma de autorizar el asilamiento y la reclusión. Desde luego, en los casos en que había «duda acerca de la locura o demencia», se hacía reconocer al individuo por peritos. Esta especificidad que limitaba la acción policial a favor de la medicalización fue mucho más clara en el código de 192737 . El médico hacía su presentación.

La autoridad médica en tanto que dispositivo para establecer obligaciones, licencias y prohibiciones, anteriormente a merced de la Iglesia católica ${ }^{38}$, tuvo una expresión palpable en esta experiencia de la medicalización de los alienados mentales en Medellín desde principios del siglo XX. En esta época, el médico comenzó a tener cierta autoridad y autonomía dentro del Manicomio; pero por fuera no desplazó a la policía ni a otras autoridades reponsables de remitir personas, y establecer la caracterización de las diferentes manifestaciones de la alienación. Las historias clínicas, que en la mayoría de los casos se limitan a una ficha de ingreso y una nota de salida por muerte o mejoría,

\footnotetext{
33 SANCHEZ (.1995), pp. 15-16.

34 Cfr. GoMÉZ (2007).

35 Montagut (1997), p. 146.

36 Cfr. GoMÉz (2007).

37 Art. 281, Código de Policía del Departamento de Antioquia, Ordenanza N 12 de 1927. Medellín, Imprenta Oficial, p. 64.

38 Obregón, D. (1989), Ritos de Exclusión y mitos acerca de la contaminación y de la higiene, Colombia, ciencia y tecnología, 7 (3), pp. 13-16.
} 
conservan como diagnóstico aquel caracterizado por las autoridades remitentes, entre las que hasta 1930 no hubo médicos. Sin embargo, el médico del Manicomio se sirvió de ellos para justificar y garantizar la exclusión de los supuestos enfermos mentales del núcleo social ${ }^{39}$.

La más antigua historia clínica que se conserva actualmente en el Archivo Histórico del Hospital Mental, es muy significativa respecto de los diagnósticos utilizados para justificar el asilamiento. Leocadia Clavijo, mujer de 57 años de edad, mestiza, soltera, que hasta 1902 se desempeñaba como maestra de escuela en la población de Abejorral ${ }^{40}$, fue remitida al Manicomio el 6 de febrero de 1903 con el diagnóstico de «melancolía crónica con delirio alimentario»; como antecedentes se señalaron «penas morales», y se hizo un pronóstico de «incurable». Leocadia estuvo asilada hasta el 15 de octubre de 1920 cuando se le dio de baja por muerte. Nunca se le hizo un registro de «historia clínica» y en consecuencia, le correspondió al médico Lázaro Uribe Calad, que había asumido como médico jefe del Manicomio en 1920 hacer una reconstrucción a posteriori de los diagnósticos y las observaciones de los comportamientos de los internos. Sobre el caso de Leocadia, el día de su muerte, anotó lo siguiente: «En los últimos meses el estado ansioso predominó. Al fin, infecciones de la piel la redujeron a la cama. A consecuencia de esto se presentaron escaras sangrado y trocanterismo y murió por caquexia infecciosa» ${ }^{41}$.

La observación sugiere el tratamiento. El proceso hacia la caquexia infecciosa podría tener dos causas: aquella que produce el estado depresivo y la falta total de apetito, presente en la mayoría de los pacientes ${ }^{42}$; pero también podría ser provocada por la aplicación muy común de los abscesos de retención, con inyecciones de trementina; tratamiento muy doloroso que impedía cualquier movimiento y reducía al interno a la cama ${ }^{43}$. Como puede verse, la observación sobre la enfermedad mental tenía sus límites en el cuerpo del paciente.

Sólo a partir de 1920, con la llegada a la dirección del Manicomio Departamental del médico Lázaro Uribe Calad, podrá hablarse en Medellín del nacimiento de un discurso psiquiátrico lo que marcó una clara diferencia entre

39 Cfr. Gómez, M. (2007), Sistematización del Archivo de historias clínicas del Hospital Mental de Antioquia, HOMO, 1903-1964.

40 Abejorral, población de la «Colonización Antioqueña», actualmente a cuatro horas al suroccidente de Medellín.

41 Archivo Histórico (HOMO), Historia clínica, No 1, 1920.

42 GómeZ, M. (2007).

43 Sobre estos tratamientos cfr. Rosselli, H. (1968), Historia de la psiquiatría en Colombia, Bogotá, Horizontes. 
la Casa de Alienados Mentales, el Manicomio y el Hospital Mental. El doctor Uribe comenzó a confrontar los «diagnósticos» de las cartas de remisión y recomendación, con los comportamientos y las observaciones hechas por la mirada médica al interior del Manicomio.

En el caso del paciente Luis María Montoya, mulato, de 27 años de edad, soltero de profesión agricultor, remitido desde el municipio de Andes ${ }^{44}$ el 4 de noviembre de 1920, con el diagnóstico de «traumatismo en el cráneo»; al ser atendió por Uribe, se puso en evidencia la necesaria observación médica practicada durante el tiempo de asilamiento en el Manicomio. Uribe había traducido «traumatismo en el cráneo» por «psicopatía traumática», pero se reservó la declaración de cualquier pronóstico que comprometiera la ética médica, señalando que «a pesar de lo dicho en el certificado, no presenta durante su permanencia aquí, ninguna de las manifestaciones aludidas» ${ }^{45}$.

A la salida del paciente, el 7 de diciembre de ese mismo año, Lázaro Uribe anotó en la ficha de registro la siguiente observación: «Desde su llegada se mostró calmado (...), guardó toda clase de conveniencias — buen juicio, buena memoria, buen fondo moral- mejorado somático y psíquicamente, fue dado de baja (...). Por mejoría ${ }^{46}$.

Con la presencia de este nuevo médico en la dirección del Manicomio, variaron el aseguramiento, el diagnóstico clínico y la curación de los pacientes. Sin embargo, fue sólo a partir de 1930 cuando la fuerza de la policía para el «mantenimiento» de los enajenados comenzó a ser desplazada por el diagnostico científico y el uso de terapéuticas especializadas propias de la práctica médica, como la administración del «sedante intravenoso»y los «electrochoques»» ${ }^{47}$.

\section{EL DISPOSITIVO}

En el año 1875, cuando Recaredo de Villa era presidente del Estado Soberano de Antioquia, se inició una campaña para recoger de las calles de Medellín a los alienados mentales. Se hizo una colecta, cuyo resultado fue de $\$ 7.416$ pesos $^{48}$, y con el apoyo de la Junta Suprema del Hospital del Estado se

44 Municipio de producción cafetera, situado actualmente a cuatro horas al suroeste de Medellín.

45 Archivo Histórico (HOMO), 1903-1987, Historia clínica ํ 19, 1920.

46 Ídem.

47 Archivo Histórico (HOMO), 1903-1987.

48 Latorre, L. (1934), Historia e historias de Medellín, Medellín, Imp. Departamental, p. 11. 
intentó fundar una casa para recluir a los alienados mentales de la ciudad.

El funcionamiento de esta casa, fue realmente muy incierto. El historiador local Latorre Mendoza ${ }^{49}$ le reconoce vida de tres años hasta 1878 cuando, de manera formal, la corporación municipal dispuso la creación de un Hospital para Locos con un personal de planta permanente ${ }^{50}$. Sin embargo, la historiadora profesional Claudia Montagut Mejía se inclina por creer que se trató de un intento fallido, pues los dineros recaudados entre los particulares, dice, se destinaron a la guerra y la atención a problemas de salud pública ${ }^{51}$.

Cualquiera que sea la fecha de comienzo del funcionamiento de la institución, lo que sí se ha determinado históricamente es que en 1878 se nombró al médico Tomas Quevedo Restrepo como director de la Casa de Alienados, y a la señora doña María Jesús Upeguí como administradora. Además, para esa fecha era muy claro el compromiso de la corporación municipal para entregar un aporte de $\$ 0.15$ pesos diarios por cada loco allí asilado ${ }^{52}$.

Con la seguridad de estos recursos, se buscaron los primeros pacientes y se hallaron en la cárcel municipal ${ }^{53}$, a donde anteriormente eran llevadas todas aquellas personas que deambulaban «sin sentido» por el espacio urbano y recibían el mismo trato que los delincuentes detenidos; pues hasta la creación del manicomio, el tratamiento a estas personas era simplemente punitivo; sin embargo, por estar detenidos «de cuenta del municipio», resultaron ser los primeros favorecidos con tal determinación. Por eso cuando se resolvió su traslado a la Casa de Alienados, la corporación municipal que en 1878 presidía Alejandro Barrientos, aprobó lo siguiente: «Páguese a la Casa de Enajenados las cuotas que determina el presupuesto vigente, una vez que pasen a ella los dementes que hoy existen en la cárcel que son de cuenta del Distrito y desde el día en que entren en aquel establecimiento» ${ }^{54}$.

De cualquier manera, la tarea de hacer funcionar una institución para el asilamiento de los locos en Medellín, estuvo sesgada por los recursos económicos, y la municipalidad se dispuso a acoger a los locos de la ciudad de Me-

\footnotetext{
49 LATORRE, L. (1934), p. 12.

50 Archivo Histórico Medellín (AHM) (1878), Ordenanzas, T. 10, pp. 2 y 51.

51 Londoño, J.B. y Uribe ANgel, M. (1933), Manicomio Departamental: Documentos históricos, Repertorio Histórico, XII (123), p. 39. Citado por MonTAGUT, C. (1997), p. 155.

52 Atendiendo a la cronología de la historia oficial, la administración actual del Hospital Mental de Antioquia, como Empresa Social del Estado, conmemorará en 2008, los 130 años de funcionamiento.

53 (AHM), Actas del Concejo (1878). T. 30, pp. 2, 16 y 51. Acuerdos (1878), T. 2181, abril, fol. 136.

54 (AHM), Alcaldía (1878), Correspondencia General, T. 17.
} 
dellín, pero resistiendo a los llegados de otros distritos o que no estuvieran cobijados por el artículo 411 del Código de Policía de 187855. Tampoco a aquellos cuyos parientes pudieran contribuir a su subsistencia.

En esta perspectiva se entiende la solicitud hecha por Alejandro Barrientos al jefe municipal, para que trasladaran a los municipios de origen, a todos aquellos locos que no recibían ningún tipo de ayuda de sus respectivos jefes municipales, con el argumento de que aquéllos no contribuían con la casa de enajenados de Medellín, y que el tesoro de esta ciudad estaba en situación crítica $^{56}$.

La intención de austeridad buscaba ahora diferenciar al loco y demente del vago o el borracho que eran excluidos de ese insipiente sistema de protección social. Sin embargo, la influencia de las demandas de atención, con las permanentes remisiones de pacientes desde los diferentes municipios del Departamento de Antioquia, hizo de esta primera casa para el asilo de los alienados mentales de la ciudad una institución de carácter regional.

Para aquella época, Medellín vivía ya una situación delicada, pues su crecimiento urbano atraía a muchos enajenados de otros distritos. En 1885, según el informe de Elías Upeguí - síndico de la Casa- de todos los locos allí asilados, apenas la mitad eran de Medellín y el resto provenía de otros distritos o con familiares que les ayudaban económicamente. De acuerdo con la resolución de la corporación Municipal, el Distrito de Medellín debería hacerse cargo únicamente de $50 \%$ de los enajenados, pero en la práctica atendía a todos los asilados, y recibía de los particulares únicamente las ayudas de los familiares pudientes. En algunas oportunidades se hicieron excepciones como en el caso de Epifanio Mejía ${ }^{57}$, poeta que fue recluido en el Manicomio de Medellín en 1878, a la edad de cuarenta años, y permaneció ahí hasta el día de su muerte, en 1913; según el doctor Jaime Mejía, quien lo visitara en el

55 CODIGO DE POLICÍA (1878). El Artículo 411 del título X Beneficencia, cobijaba a los niños desamparados, adultos sin domicilio, mendigos, locos y dementes. El título V Moralidad y Buenas costumbres, señalaba la ociosidad, la embriaguez y la prostitución como objetos punibles en el código. Cf. Montagut, C. (1997), p. 142.

56 (AHM), Alcaldía (1878), Correspondencia General, T. 17.

57 Epifanio Mejía (1838-1913). Destacado personaje de las letras en la ciudad de Medellín a finales del siglo XIX. Nacido en la población de Yarumal al norte de Antioquia, su familia se desplazó a la ciudad de Medellín, atraída por el auge de la economía comercial. Fue escritor y poeta, y publicó en importantes revistas de la época como El Oasis. Es el autor del Himno Antioqueño. Por su prolongado asilamiento, su nombre ha permanece ligado al de la historia del Hospital Mental. Cfr. Rosselli, H. (1987), La locura de Epifanio y otros ensayos. De historia, medicina, psiquiatría y psicoanálisis, Bogotá, Ediciones Tercer Mundo. 
manicomio en 1882 , «víctima de una cruel melancolía» ${ }^{58}$. A solicitud de familiares del poeta y en atención a sus méritos como compositor y escritor, se le concedió por resolución del jefe municipal, la exoneración de todos los costos de su asilamiento.

En los códigos de policía, entre 1878 y 1914 se mantuvo una constante:

No se podrá obligar a la familia del loco o demente a tenerlo en su hogar; por otro lado, la policía tiene el poder de compeler a la persona que crea deba correr con los gastos, con multas o mediante el poder judicial (Código de policía 1914, Art. 166 y 168$)^{59}$.

En ambos códigos se dispuso arresto en primera instancia, pero sólo en el código de 1914 aparecía el hospital en el artículo del capítulo locos y dementes.

El artículo 170 del código de 1914 expresaba lo siguiente:

(...) cuando en el municipio donde aparezca un loco o demente no hubiere establecimiento alguno apropiado para tenerlo en seguridad, el jefe de policía ordenará que sea conducido al manicomio del departamento. Los gastos de conducción se harán por cuenta de los deudos del loco o demente, si estos pudieran hacerlo; o en caso contrario del municipio de donde procede ${ }^{60}$.

Una proporción de la procedencia de los internos en la casa de enajenados mentales en la fecha de su primera fundación se puede apreciar en el siguiente cuadro:

LOCOS ASILADOS EN LA CASA DE ALIENADOS MENTALES DE MEDELLÍN EN 1885

\begin{tabular}{|l|c|c|c|c|c|}
\hline Sexo & \multicolumn{3}{|c|}{ Procedencia } & Con familia & Total \\
\hline & Ignorada & Medellín* & Otros distritos & & \\
\hline Hombres & & 6 & 3 & 7 & 16 \\
\hline Mujeres & 1 & 9 & 1 & 2 & 13 \\
\hline & 1 & 15 & 4 & 9 & 29 \\
\hline
\end{tabular}

* Se incluye en los de Medellín, también a los de las fracciones de Belén y el Poblado.

Fuente: Archivo Histórico de Medellín (AHM), Alcaldía (1885), T. 17.

58 MeJía, M.J. (1960), Historias médicas, Medellín, Editorial Bedout, p. 68. Citado por RosselLi (1987), p. 21.

59 Montagut, C. (1997), p. 147.

60 Artículo citado por MonTAgUt (1997), p. 147. 
Resulta importante señalar que la acción de la municipalidad de Medellín sobre el asunto de los enajenados mentales no es simplemente cuestión de civismo o la mera búsqueda de una estética urbana. Con ello, se estaba expresando la emergencia de un nuevo dominio de medicalización en la ciudad de Medellín. La intervención de la medicina como instrumento de control social se enfocaba a la regulación de los comportamientos, a la delimitación de los mismos, y a la separación de los alienados del cuerpo social.

Entre finales del siglo XIX y comienzos del XX la medicina adquirió un matiz social. Ya no se trataba de la relación entre un paciente, la enfermedad y las terapéuticas curativas, sino de la relación con el espacio público. Para ello no solamente se hizo necesaria la transformación de las estructuras urbanas en aras de la salubridad pública, sino que se reconsideraron las prácticas frente a los alienados mentales y otros personajes de la anomia de la ciudad (ancianos, niños huérfanos, vagos, prostitutas, mendigos). Se trataba de la expresión de la medicina social en tanto que medicina urbana.

En el ámbito de la ciudad de Medellín, la medicina social y la transformación de las formas de tratamiento en las enfermedades mentales, evolucionó desde la exclusión coercitiva hacia la clasificación de los enajenados, mediante un insipiente registro clínico y su separación de los enfermos no mentales y de los delincuentes, con miras a aplicar una terapia curativa basada en el asilamiento. Se dio entonces un sentido distinto a las formas de control social heredadas desde la Colonia por la ciudad republicana, que atendían simultáneamente y sin distinción al conjunto de «necesidades básicas» que abarcaban el cuidado del cuerpo, de la moral y del alma ${ }^{61}$.

Podría decirse entonces, que la medicina social apareció en Medellín como producto del crecimiento de la ciudad. Para fines del siglo XIX, cuando los procesos de urbanización se vieron acompañados por la presencia notoria de locos; aumentó también la preocupación de la municipalidad por ejercer un control sobre los llamados enajenados mentales. La demencia fue considerada una enfermedad que debía ser tratada de forma especializada, por cuenta de instituciones estatales, con la colaboración de los particulares y las comunidades religiosas, para lograr la máxima eficacia. La acción de esa «medicina urbana» sobre las enfermedades mentales, llevó a una rápida evolución de los conceptos y formas institucionales de tratamiento; aunque en un comienzo se hubieran aplicado típicas formas de la «medicina de exclusión» ${ }^{62}$.

61 Cf. Vargas, J. (1988), Santa Fe y Bogotá: Evolución histórica y servicios públicos. Bogotá 450 años. Retos y realidades, Bogotá, Foro Nacional por Colombia e Instituto Francés de Estudios Andinos, p. 11.

62 Cfr. Foucault, M. (1977), Historia de la medicalización, Educación médica y salud, 11 (1). 
Así, en 1875 nació la Casa de Alienados; la misma que en 1878 y bajo la dirección del médico Tomás Quevedo Restrepo, sería el Hospital de Locos ${ }^{63}$; después el Manicomio, por ordenanza de 1888; y en 1892, bajo la dirección de Ricardo Escobar Ramos, pasaría a un edificio especialmente diseñado para el aislamiento de los enajenados mentales o locos, y al que se conocería con el nombre de «Manicomio de Bermejal» ${ }^{64}$. Con este nombre funcionó, con ocho directores consecutivos, hasta 1951, cuando fue creado el Hospital Mental de Antioquia, con programas integrales para el tratamiento de los enfermos allí internados. A diferentes nombres, correspondieron momentos conceptuales distintos de la medicina que se ocupaba de las enfermedades mentales en el medio urbano.

En un primer momento, los locos estuvieronn incluidos - al lado de los mendigos, los pobres de solemnidad, las mujeres de «vida desordenada», las viudas sin familia, los niños huérfanos, las personas afectadas por enfermedades contagiosas y los viejos - entre las personas a quienes iba dirigida la caridad cristiana y la asistencia social. Pero con el desarrollo de la ciudad moderna, el tratamiento para ellos cambió y se establecieron instituciones especialmente destinadas a darle una solución social a la presencia de los dementes ${ }^{65}$. La demencia exhibida o callejera, se convirtió en un problema social urbano y, en consecuencia, dejó de estar regulada por la caridad, es decir, por el azar, y comenzó a ser codificada y luego medicalizada.

En 1914, el Manicomio funcionaba como una institución del Departamento de Antioquia y asilaba no sólo a pacientes de Medellín, sino de otros municipios. Estaba regido por la Junta del Manicomio, que a su vez dependía de la Junta de Higiene del Departamento, cuyos programas dependían de la Asamblea Departamental y la Gobernación. Ese año, se produjo la segunda reglamentación del Manicomio, desde que la Gobernación lo asumió como regio-

63 (AHM), Acuerdos (1878), T. 2181, fol. 136.

64 Montagut, C. (1997), p. 156.

65 Otras experiencias de medicalización de la locura, muestran este proceso institucional hospitalario, cf. PATLAN, M.E. (1997), El hospital para hombres dementes: San Hipólito en el siglo XIX. En ArboledA, L. y Osorio, C. (eds.), Nacionalismo e internacionalismo en la historia de las ciencias y la tecnología en América Latina, Calí, Universidad del Valle, 217-222. También, CARBAJAL, A. (2001), Mujeres sin historia. Del Hospital de La Canoa al Manicomio de la Castañeda, Secuencia: Revista de historia y ciencias sociales, 51 (sep.-dic.), pp. 31-56. SACRISTÁN, C. (2005), Por el bien de la economía nacional: trabajo terapéutico y asistencia pública en el Manicomio de La Castañeda de la ciudad de México, 1929-1932, História, Ciências, Saude-Manguinhos, 12, 675-692. AmARANTE, P. (1994), Uma aventura no manicômio: a trajetória de Franco Basaglia, História, Ciências, Saude-Manguinhos, Out, 1 (1), 61-77. 
nal en 1892, en el sitio de Bermejal. La primera la habían hecho en el año 1896, los doctores Eduardo Zuleta y Juan B. Londoño, comisionados por la Academia de Medicina, por encargo directo del gobernador del Departamento $^{66}$. La presencia de la sociedad científica no era incompatible con la participación de las religiosas en la administración del manicomio, en el reglamento de 1914, ya que éste definía lo concerniente a la administración del establecimiento en manos de laicos y de religiosas de la siguiente manera: «Todos los empleados [del Manicomio] subalternos, exceptuando las hermanas de la caridad y los que de ellas dependen, deben ser nombrados y removidos por el Director de la Junta del Manicomio» ${ }^{67}$.

En esta materia era evidente la armonía de instituciones médicas civiles con las comunidades religiosas, en la administración del control y tratamiento de los enfermos mentales de la ciudad.

En el Reglamento, el Distrito señaló que las bases para la internación de los asilados serían la «Ley Francesa de junio de 1838 y la Ordenanza de la Asamblea Departamental de 1839 en lo que sea adaptable» ${ }^{68}$. El sistema de organización impuso también un registro de las actividades administrativas en varios libros que daban información sobre el movimiento de los pacientes en el hospital, las prescripciones sobre cada enfermo, los inventarios y la contabilidad.

Desde el punto de vista médico, el reglamento de 1914 expresó una filosofía que proponía la utilización de «métodos de libertad» antes que formas coercitivas o de fuerza. Se recomendaba igualmente la implementación de «tratamiento hidroterápico» y las más estrictas «medidas de higiene», sobre todo en la prevención y control de moscas y parásitos como niguas, chinches y pulgas. Se definieron también las bases para la alimentación de los enfermos mentales, y la separación entre éstos y los afectados por enfermedades no mentales, como las contagiosas, y en general su separación de las personas con las que inicialmente compartían su aislamiento. De esta idea de la separación es que surgió la necesidad de construir un pabellón exclusivo para los enfermos mentales y su tratamiento en Medellín.

Por otro lado, el reglamento contempló también la actividad de los internos dentro del proceso de tratamiento y asilo. Se recomendó entonces mante-

66 (AHM) (1896), Informes, Junta Departamental de Higiene, pp. 52, 53, 54.

67 Reglamento del Manicomio Departamental (1914). En Gaceta Departamental, Medellín, 13 de agosto, p. 41.

68 (AHM) (1839), Ordenanzas. Acerca de la experiencia francesa, cf. PERROT, M. (1991), Sociedad burguesa: aspectos concretos de la vida privada. En Historia de la vida privada, T. 8, Madrid, Taurus, pp. 131-139. 
nerlos trabajando en talleres, y ocuparlos en oficios domésticos, el cultivo de jardines y árboles, y la agricultura en general. En materia de recreación, se recomendó en este reglamento la proyección de películas y sesiones con gramófono, pues se aseguraba: «La música tiene una feliz influencia en estas enfermedades $\rangle^{69}$.

Las bases generales de este reglamento mostraban una clara orientación clínica para el control de los enfermos mentales de todo el Departamento desde el Manicomio, el cual estaba regido por cuatro instancias administrativas: la Junta del Manicomio, la Junta de Higiene, la Asamblea Departamental y la Gobernación de Antioquia.

A juzgar por una noticia divulgada en el periódico local La Defensa de 1937, el reglamento de 1914 se había hecho inoperante: al parecer, el manicomio continuaba siendo en muchos aspectos un lugar de aseguramiento de los alienados y no un espacio para el tratamiento clínico, y el aumento considerable de la población de asilados exigía cambios. Según esa noticia la población de asilados había aumentado a alrededor de quinientas personas, las cuales permanecían «encerradas en jaulas» ${ }^{70}$.

La noticia anunciaba además la implementación de variaciones en los mecanismos de regulación de la institución. La toma de decisiones se dejó en manos del director del Manicomio, que a partir de ese año fue designado médico-jefe. El nuevo reglamento señaló que ningún enfermo podría entrar ni salir sin el permiso del director, que debía ser un médico. Además, el servicio médico, en todo lo concerniente al régimen físico y moral, y a la «policía médica y paramédica», quedó también a cargo del director. La concentración de funciones y responsabilidades fue tal, que la misma reforma al reglamento contemplaba la residencia del médico jefe dentro del Manicomio. Otro aspecto importante de la reglamentación de 1937 es que no incluyó nada sobre la participación de las comunidades religiosas en el proceso de la administración del hospital o de la atención a los enfermos.

En 1937 se reafirmaron las funciones de la «Junta de Inspección y Vigilancia del Manicomio», creada por la Ordenanza 25 de 1914 y compuesta por el gobernador del Departamento, los miembros de la Junta Departamental de Higiene y el médico jefe del Manicomio ${ }^{71}$.

69 (AHM) (1914). Ordenanza Departamental 25. Junta de Inspección y Vigilancia del Manicomio. «Reglamento del Manicomio Departamental». En Gaceta Departamental, Medellín, 13 de agosto, p. 15.

70 La Defensa, nov. 17 de 1928, citado por BetanCuR, (2000), p. 113.

71 Ibíd. 
Se tiene así, que la regulación de la salubridad pública, en materia de enfermedades mentales, pasó en Medellín de la municipalidad al Departamento, pues más allá del control social sobre los vecinos de Medellín, el Manicomio se vio obligado a recibir a muchos alienados, locos o dementes que llegaban de otras regiones del Departamento. Podría decirse entonces, que en 1892, apenas a 17 años de la creación de la Casa de Alienados de Medellín como una expresión de la forma de la «medicina urbana», las demandas que acosaban a la capital del Departamento y la presión de las migraciones desde otros distritos, convirtieron esta clínica para la atención en la ciudad en receptora de los enajenados del Departamento y en centro de atención médica en el nivel regional. Se aprecia desde esta perspectiva, un crecimiento de las demandas regionales y urbanas por la salud mental, y el aumento de la capacidad medicalizadora con forma de aislamiento centralizado en la ciudad de Medellín.

Uno de los factores que hicieron posible la centralización de la medicalización de las enfermedades mentales, fue la existencia de la Academia de Medicina de Medellín y de la Escuela de Medicina de la Universidad de Antioquia. La primera, como se dijo arriba, formuló el reglamento de 1896, como expresión del peso y la influencia que el cuerpo médico, organizado en sociedad científica, tenía en la municipalidad, en su función de regulador de la salud pública en Medellín y el Departamento. Las políticas públicas en materia de salud estaban mediadas también por la medicalización que se ejercía tanto desde la Universidad, por medio de la Clínica de Enfermedades Mentales, cátedra fundada en la Escuela de Medicina de la Universidad de Antioquia en cumplimiento de la Ordenanza 25 de 1914, y que fue proyectada directamente en el Manicomio, en la medida en que tenía como responsable a su director ${ }^{72}$. De esta manera, el director del Manicomio estaba comprometido con una instancia importante de la regulación de la salud mental, que incluía la formación de nuevos médicos especializados, y de la investigación. La dedicación del director del Manicomio a la Clínica de Enfermedades Mentales fue reafirmada en 1915, cuando la Ordenanza 51 de ese año creó nuevos cargos en el Manicomio. Se nombró un médico auxiliar y se creó el cargo de síndico-contador; se conservó a las hermanas de la presentación de Tours para el cuidado del establecimiento y de los pacientes, merced que les había dado desde 1905 el gobernador Teodonio de Villa, cuando quedaron encargadas de la «dirección interior del establecimiento, particularmente en los referente al buen orden, aseo y a la moralidad y vigilancia de los sirvientes $\rangle^{73}$.

72 Ibíd.

73 (AHM) (1915), Ordenanza 15. 
Una prueba de esta participación religiosa en las funciones de regulación en el cuidado de la salud, se ve en la descripción que Juan B. Londoño hace del Manicomio en 1906, en la que destaca muy especialmente «la función cumplida allí por las hermanas de la caridad $\rangle^{74}$. Hasta 1913 , cuando se alcanzó una mayor identificación de los propósitos clínicos del aislamiento, las funciones del director se limitaban a las de «simple médico»; mientras que las funciones de dirección y administración del establecimiento quedaban en manos de las monjas y otros empleados. Durante esos años no se produjeron registros clínicos.

Desde su emergencia en la ciudad de Medellín, el Manicomio se organizó desde la iniciativa de la municipalidad o de la Gobernación, niveles estatales que regulaban la salud y la higiene pública. Sin embargo, las iniciativas religiosas se involucraban siempre con las actividades asistenciales y de salud; concretamente, como ya se mencionó arriba, para el caso del Manicomio, era clara la participación de monjas que cumplían funciones de cuidado, higiene y recreación dentro de los programas reglamentarios. En el nivel administrativo, frente a la regulación oficial, sociedades de un definido corte religioso, como la San Vicente de Paúl, estuvieron atentas, a finales del siglo XIX, para tomar por su cuenta las riendas del Manicomio. Como producto de esta participación, en 1898 se suspendieron los contratos con el síndico y la directora; y se celebró un nuevo contrato, para la administración del Manicomio, con el doctor Escobar Ramos, presidente de la Sociedad San Vicente de Paúl ${ }^{75}$.

Esta conservación de la intervención religiosa en la administración, hasta mediados del siglo XX, marcó lo religioso como factor delimitador de la locura al lado del médico. Sin embargo, hasta 1952, la marginación que causa el encierro, no produce un verdadero aislamiento de los dementes con respecto a la ciudad. La cercanía administrativa del Manicomio con la cárcel y su ubicación en el mapa de la ciudad, expresaba aún la integración de los cuerpos. Una de las primeras gestiones administrativas de la casa para locos en 1878 fue el préstamo de cadenas para amarrar a los locos, solicitadas a la dirección general del presidio ${ }^{76}$. Igualmente los miembros del Consejo de la ciudad dispusieron la construcción de tapias de altura para la «seguridad e independencia de los edificios» ocupados por el hospital de $\operatorname{locos}^{77}$.

\footnotetext{
74 (AHM) (1906), Junta de Inspección y Vigilancia del Manicomio, p. 55.

75 (AHM) (1898), Actas y Asuntos Varios, T. 33, pp. 52, 53, 54.

76 (AHM) (1878), Tomo 2181, 23 junio, fol. 436. Citado por MonTAGUT, C. (1997), p. 157.

77 (AHM) (1892), Tomo 2181, 26 oct., fol. 282. Citado por MonTAGUT, C. (1997), p. 158.
} 
Sólo en los informes del médico Juan B. Londoño comenzó a hablarse de curación de la locura desde la medicina, con la exclusión social como terapia.

La descripción del médico Juan B. Londoño - que fue director del Hospital en dos ocasiones, de 1902 a 1904 y de 1914 a 1917 -, exaltó la calidad del Manicomio Departamental y lo comparó con el Manicomio de Parra de Bogotá y con el de Santa Ana de París. Un buen Manicomio, decía Londoño, debía incluir la cirugía entre sus servicios pues muchas de las «locuras» tenían su origen en daños orgánicos curables.

Después de los medios físicos: aire puro, luz abundante, hidroterapia, electroterapia y ejercicio (trabajo) y buena alimentación; viene la cirugía a perfeccionar el tratamiento de la locura, pues, como lo ha demostrado con multitud de hechos Lucien Piaqué (Doctrinas y Hechos) en la generalidad de las locuras esenciales, el órgano menos afectado es el cerebro (...) para hacer un buen Manicomio, (...) debe dotarse de los elementos que tiene el simpático Asilo de Santa Ana de París; y hacer en otro lugar adecuado la granja agrícola, sin la cual no se puede curar la mayoría de los $\operatorname{locos}^{78}$.

Esta referencia a la practica de la cirugía, y al ambiente de granja agrícola en la búsqueda de una solución médica para los enajenados, significaba el surgimiento de una nueva forma de intervención médica; aquella que desplazaba el pensamiento religioso y la intervención policial. Una práctica más científica, en tanto que reconocía el saber y la experiencia de la medicina; si bien será sólo a partir de 1920, con el médico Lázaro Uribe, una experiencia en el campo de la psicopatología.

Fecha de recepción: 24 de septiembre de 2007.

Fecha de aceptación: 5 de mayo de 2008.

78 (AHM). LONDOÑO, J.B. (1914), Correspondencia, pp. 55, 56, 57. 